\title{
Modelling of mitigation of the power divertor loading for the EU DEMO through Ar injection
}

\author{
Fabio Subba \\ NEMO Group, Dipertimento Energia, Politecnico di Torino, C.so Duca degli Abruzzi \\ 24, Torino 10129, Italy \\ E-mail: fabio.subba@polito.it
}

Leena Aho-Mantila

VTT, TI-02044 Espoo, Finland

\section{David Coster}

Max Planck Institut fuer Plasmaphysik, Boltzmannstrasse 2, 85748 Garching bei Muenchen, Germany

Giorgio Maddaluno

ENEA Centro Ricerche Frascati, Frascati, Italy

\section{Giuseppe F. Nallo}

NEMO Group, Dipertimento Energia, Politecnico di Torino, C.so Duca degli Abruzzi 24 , Torino 10129, Italy

\section{Bernard Sieglin}

Max Planck Institut fuer Plasmaphysik, Boltzmannstrasse 2, 85748 Garching bei Muenchen, Germany

\section{Ronald Wenninger}

Max Planck Institut fuer Plasmaphysik, Boltzmannstrasse 2, 85748 Garching bei Muenchen, Germany

\section{Roberto Zanino}

NEMO Group, Dipertimento Energia, Politecnico di Torino, C.so Duca degli Abruzzi 24, Torino 10129, Italy 
August 2017

\begin{abstract}
We present a computational study on the possibility to mitigate the divertor load problem for the EU DEMO through impurity injection, done with the SOLPS code. We consider only power crossing the separatrix level corresponding to $\mathrm{H}$-mode operation, and define the machine operating condition through the upstream electron densities and impurity level. This study focus on divertor conditions, so that we select Ar as the injected impurity, based on its high radiation efficiency at SOL characteristic temperatures. We monitor target conditions for different operational points, considering acceptability threshold determined by $\mathrm{W}$ sputtering level for the electron temperature and cooling system capacity for the peak heat flux. Our simulations suggest that it should be possible to operate DEMO in divertor-acceptable conditions, but the upstream density should be at least close to $50 \%$ of the Greenwald limit, so that the available operational window may be narrow
\end{abstract}

Submitted to: Plasma Phys. Control. Fusion

\title{
1. Introduction
}

The EU DEMO is currently in its conceptual design phase, where a number of fundamental decisions have to be taken on the overall project, which will direct many future efforts. Most parameters of the machine are still to be set, trying to optimize the production of electrical power in an economically attractive and reliable way. It is widely acknowledged that the power exhaust problem is a potential showstopper for the development of first a prototype and later a commercial fusion reactor [1]. The baseline strategy foreseen for its solution relies on the development of high-radiative scenarios, which have already been obtained on all metal machines [2]. The main characteristics of this configuration are the choice of fully metallic chamber ( $\mathrm{W}$ for DEMO), and the injection of a suitable amount of impurities to radiate the large majority of the power entering the Scrape-Off Layer, to take advantage of the extended wall surface area to reduce the peak power load density down to a tolerable level [3]. The successful realization of such program requires a number of choices to be made. Among the others, the radiating impurity to be puffed should be selected. The chosen impurity should guarantee the dissipation of $90 \%$ or more of the input power [3] and, at the same time, be compatible with core plasma conditions. This paper concentrates on a first sub-step of the overall strategy, analysing the possibility to reach acceptable divertor conditions by means of a single impurity radiator introduced in the plasma. For the time being, this study leaves out important questions like, for example, compatibility of the setup studied with the main plasma conditions. Consequently, we are not aiming at presenting a fully self-consistent solution of the power exhaust problem in DEMO; instead, we want to understand if such a solution is, at least in principle, possible with the framework of a conventional Single Null divertor, and which criticalities may be expected along this path. 
The paper is structured as follows. In the next section, we discuss in more detail the overall aim of our work and the methodology we adopted to pursue it. Next we introduce the numerical setup of our modelling. We give some details on the physics we decided to include in our modelling trying to balance numerical accuracy and computational resources requirements, and briefly discuss the mesh chosen for this work, giving a tentative assessment of the uncertainty of our results due to the finite mesh-size. The main section of the paper follows, in which we present and discuss our results. The paper closes with a brief comment on our findings and on the points still left open.

\section{Aim and methodology}

In this paper we focus on the possibility to operate the EU DEMO in an acceptable way in terms of the divertor integrity, which will need to tolerate steadily a very aggressive environment. The exact value of the safe operational limits are not precisely known, but considerable efforts are ongoing to produce estimates as accurate as possible [3], [4]. As far as target conditions are concerned, there are essentially two main constraints we should account for. On one side, the peak heat flux on the target $q_{\text {max,tar }}$ should not exceed the cooling system capability to keep the solid surfaces within their acceptable temperature window. For current cooling technology and target material choice (W) a reasonable estimate of the maximum tolerable value is $q_{\max , t a r}<10 \mathrm{MW} / \mathrm{m}^{2}$ [3]. The second constraint arises from the need to limit sputtering of $\mathrm{W}$ atoms, which could accumulate in the core plasma and shut down the discharge through excessive radiation. The most effective sputtering producers are highly charged heavy impurity ions, which can acquire a significant amount of energy by falling through the $\approx 3 T_{e}$ potential across the electric sheath. In order to limit the process we should then keep the target electron temperature sufficiently low. An accurate evaluation of $T_{e, \max , t a r}$ limit must consider the nature of the projectile ions and divertor material. It has been estimated that, for $\mathrm{Ar}$ impurities impinging on $\mathrm{W}$, a reasonable limit could be $T_{e, \max , \operatorname{tar}}<5 \mathrm{eV}$ [3]. In addition to the above constraints involving the target conditions, the DEMO SOL should fulfil few further requirements. In order to guarantee $\mathrm{H}$ mode operation, a minimum amount of power should be conducted/advected into the SOL through the separatrix. Following [5], we take as reference value $P_{\text {sep }}>150 M W$. Finally, the SOL electron density should be a not too large fraction of the Greenwald limit, which for DEMO can be estimated to be $8.6 \times 10^{19} \mathrm{~m}^{-3}[3]$.

In order to help identifying possible DEMO regimes compatible with such strict divertor requirements we built a database of SOLPS simulations, trying to cover a reasonably large subset of SOL parameters. Since in DEMO we expect to have at least $P_{\text {sep }}=150 \mathrm{MW}$, it is clear that impurity radiation will play a major role in the SOL to distribute most of such power onto the outer wall. In the present paper, we chose to consider a radiator species only in order to keep the analysis as simple as possible. Ar was selected as the desired impurity, because of its chemical compatibility with $\mathrm{W}$, its capacity to limit sputtering provided the plasma temperature in front of the target is 
kept below the threshold of $5 \mathrm{eV}$, (which should happen in detached target operation), and its relatively large radiative power at SOL-typical temperatures [6]. Figure 1 shows the line radiation rates for all the species considered in our simulations as a function of $T_{e}$, taken at a reference electron density $n_{e}=2 \times 10^{19} m^{-3}$. Among all the species considered, the neutral ones $\left(D^{0}\right.$ and $\left.A r^{0}\right)$ have relative large radiation rates. However, a considerable presence of such impurities is only compatible with relatively cold plasmas (for example $A r^{0}$ has a first ionization potential of $\approx 15.8 \mathrm{eV}$ ) which, as we will see later, are difficult to obtain in our simulations except, perhaps, very close to the target, as we will discuss below. $D^{1}$ is obviously massively present in our modelling, but for any temperature of interest here its radiating power is several orders of magnitude smaller than that of Ar, so that even a minor presence of such impurity will make its contribution to radiative losses the dominant one. $A r^{1+17+}$ is a bundled charge state combining all the Ar ionization stages except the last one (in section 3 we will discuss the choice of the bundled charge-state approach and other details of our simulations setup). Over the temperature interval of interest here, it has a radiative power much larger than $A r^{18+}$. Furthermore, the last ionization potential for $\mathrm{Ar}$ is $\approx 4.4 \mathrm{KeV}$ per atom, an amount of energy far larger than typical SOL temperatures. $A r^{18+}$ will then be present in the SOL in tiny traces only, and will not give any significant contribution to power losses. We conclude that, within the scope of our analysis, the important specie will be $A r^{1+}{ }^{17+}$ (always) and neutrals in case we can obtain sufficiently cold plasmas. In the following discussion we will focus on these species only. We assume that the SOL conditions can be globally characterized by $P_{s e p}, n_{e, O M P}$ and the Ar level. In order to limit the number of free parameters, we performed all our simulations at $P_{s e p}=150 \mathrm{MW}$. We let $n_{e, O M P}$ to vary in the range $2.5 \times 10^{19} m^{-3}-6 \times 10^{19} m^{-3}$, corresponding to $29 \%-70 \%$ of the Greenwald density, respectively [3]. This relatively large range is motivated by our lack of knowledge of the critical value of $n_{e, O M P} / n_{G W}$ above which confinement will start deteriorating. Considering data from JET and AUG we do not see a constrain for confinement when $n_{e, O M P}$ is in the range of $0.4 \times n_{G W}$ or lower [7]. The exact upper limit is under investigation; we expect a transition to L-Mode for $n_{e, O M P}$ in the range $0.4-0.5 \times n_{G W}$. As it will be clear later, the most dangerous condition would realize if the critical $n_{e, O M P} / n_{G W}$ value happened to be noticeably lower than expected. For each $n_{e, O M P}$ value, the impurity level was progressively increased, starting from a clean plasma, until either we obtained satisfactorily detached conditions or a pedestal impurity level too high to be practically relevant.

\section{Modelling setup}

The SOLPS package follows from modelling tool for tokamak edge plasmas, assuming toroidal symmetry. It implements a wealth of physics details and is highly flexible, for the user to choose the desired level of accuracy [8]. The precise setup selected in our model is a balance between the need to obtain results as realistic as possible, to keep the pace with a design which is still rapidly evolving at a relatively fast pace and to scan 


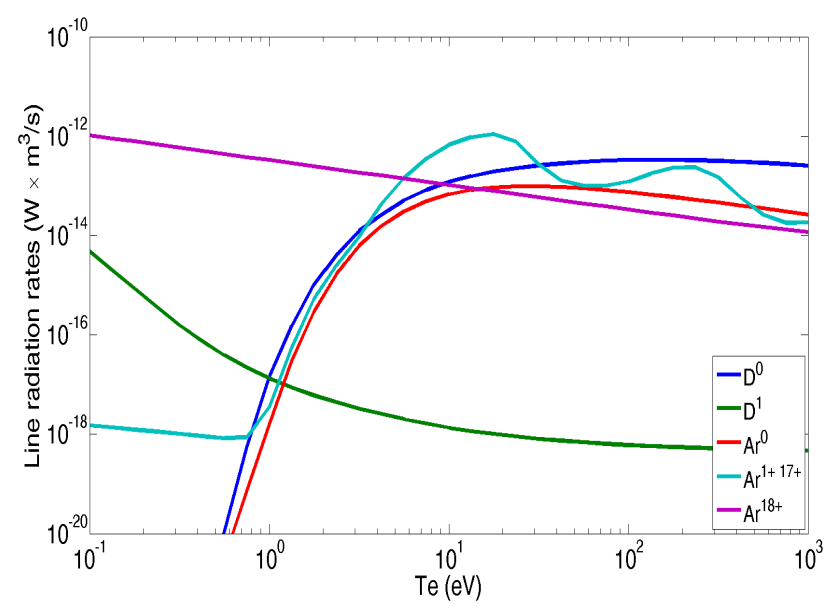

Figure 1. Radiation rates for D and Ar

a large number of possible conditions in an acceptable time. In order to meet as many of the listed requirements as possible at the same time, we took the following decisions on the physics to be included/excluded in/from our model.

a) Neutrals are obviously a major player in a detached divertor, as DEMO is expected to have. SOLPS may run the Monte Carlo solver EIRENE in order to model neutrals in the kinetic regime, or a faster fluid-neutral model. In the simulations to be presented below, we decided to adopt the second option. Assuming neutrals are in the fluid regime may obviously be a bold assumption, depending mostly on the temperature of the plasma. An initial study described in [9] suggests that such approximation may be acceptable for detached plasmas, while it is likely to be grossly wrong in attached conditions, due mostly to the larger electron temperature. However, it should be noticed that attached divertor conditions are not of interest in this paper, because it is almost impossible that DEMO will be able to operate with a steadily attached divertor. We then assume that our approximation is acceptable, at least for the cases of major interest in the following. A second drawback of adopting the neutral fluid model is that this can consider only a rough sketch of the divertor geometry (see the discussion below on the computational choices). This prevents us from making any accurate prediction on a few important details like, for example, the advantage of including (or not) a dome in the divertor, or predicting the pumping efficiency. Such details are post-poned to a future study.

b) Drifts are known to influence under certain conditions the particle and power distribution between the two divertor legs [10]. However, we are not aware of any study in which drift effects do change in a major qualitative way an edge plasma picture obtained neglecting them, although such a condition could in principle be possible [10]. Furthermore, it is a common experience that SOLPS may become unstable, or at least converge much less easily, when the user wants to consider the full drifts effects in the simulations. Based on the above considerations, we decided to neglect drifts for the time being.

c) Impurities are critical for our study, because the capacity to accurately predict 
how much power can be radiated in the SOL essentially depends on accurate impurity modelling. In principle, SOLPS can follow each ionization stage of any atomic species in the plasma as an independent fluid. However, this would generate the need to follow more than ten different fluid species, which would slow down the simulations considerably. In order to avoid this problem, we adopted the bundled charge state approach described in [11]. It is a technique to represent a set of different charge states with a single equivalent fluid, with an effective charge value depending on the local plasma conditions. In the most aggressive form adopted here, the final result is that the total number of independent fluids considered for a non-hydrogenic atomic species is three, corresponding to (i) the neutral atoms, (ii) all charge states except the fully ionized one, and (iii) the fully ionised stage. A first assessment of the accuracy to be expected from the bundling technique has been presented in [11], where it was concluded that this technique reproduces acceptably some macroscopic quantities like, e.g., global balances, while accurate representation of local details could benefit from the all-species representation. We plan switching to the all-species representation in a second phase of this activity, when we will activate the kinetic-neutral model for a few selected relevant cases.

Figure 2 shows the computational domain considered in our calculations. The actual modelled region is cyan coloured. The red lines are the target plates, and we also included a representation of the separatrix (dark blue). The domain extends about $10 \mathrm{~cm}$ within the core (measured at the OMP), aiming at representing approximately the DEMO pedestal. A drawback of purely fluid simulations with SOLPS is that the code is not able to represent the actual wall shape, which is artificially substituted by an effective wall, coinciding with a magnetic surface [8]. This affects all external surfaces except the targets. In figure 2 we added a representation of the DEMO vessel (dashed line) to help the reader evaluating the impact of the geometrical simplification. However, it should not be forgotten that our current model does not see anything beyond the coloured core and SOL portions.

The energy balance in our plasma is driven by the power injected across the separatrix as a boundary condition. We decided to fix the value $P_{s e p}=150 \mathrm{MW}$, which is a level expected to guarantee robust H-mode operation [3]. It has to be noted that the separatrix is actually a magnetic surface internal to our domain, so that setting $P_{\text {sep }}$ is not strictly speaking a boundary condition. The result is technically achieved by feedback adjusting the core boundary temperature until the desired $P_{\text {sep }}$ value is obtained. As a side effect, the input power actually entering the computational domain is larger than $150 \mathrm{MW}$, including also the pedestal radiation and Bremmstrahlung losses. In principle, this additional information could allow trying to investigate the compatibility of the SOL conditions we obtained with the core plasma. However, accurate discussion of this topic would require considering a high-Z core radiator, which is beyond the scope of the present paper. The major power sinks are obviously the target plates and the radiation induced by the injected impurities. A minor amount of power exits the domain from the wall and Private Flux Region (PFR) boundaries. However, these two regions 


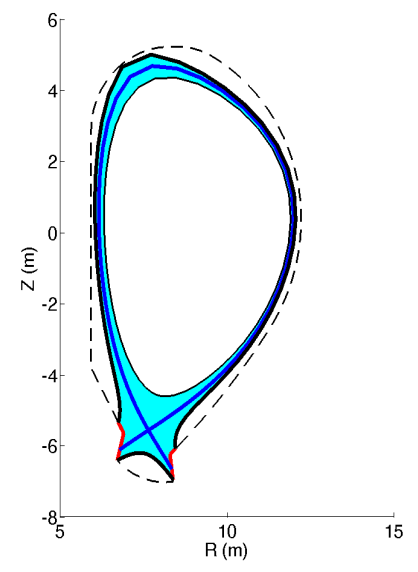

Figure 2. Computational domain used in our calculations. Red lines are the target plates. The dashed line is a sketch of the DEMO wall.

influence the total energy balance for a tiny fraction only: we will not discuss them further.

The particle balance is more sophisticated. At the pedestal boundary we let $\mathrm{D}$ ions enter the domain at a rate of $5 \times 10^{20} \mathrm{~s}^{-1}$, representing a possible small diffusive contribution from the main plasma. Most particles enter the plasma as a volumetric $D^{0}$ source located in the pedestal, mimicking pellet fuelling at a rate of $2.3 \times 10^{22} \mathrm{~s}^{-1}$ atoms/s. Operatively, we wish to control the plasma density in our domain by setting its value at the outboard mid-plane. We achieve this by puffing an additional feed-back controlled $D^{0}$ flux at the (artificial) wall boundary. D ions are fully recycled into neutrals, while particle exhaust is guaranteed by allowing a leakage flux for $D^{0}$ at the PFR.

The computational mesh choice is a further element influencing critically the balance between numerical accuracy and required computational time. We selected a $96 \times 36$ (poloidal $\times$ toroidal) mesh, which is usually considered an acceptable compromise [12]. An independent assessment of the accuracy to be expected based on a Richardson extrapolation analysis [13] suggests that results obtained on such a mesh can be acceptably accurate for detached plasma cases, much less for attached ones [14]. As already noticed, we have little interest in attached plasma conditions, which should not be relevant for DEMO anyway. Consequently, we feel safe to accept for such cases a purely qualitative description, with only marginal quantitative accuracy ambition. As already stated, the spatial distribution of the power radiated as a consequence of the interactions of the plasma with the seeded impurities is an output of the calculations. Assessing the impact of this radiated power on the overall heat flux on the target plates is important since its contribution may be significant, depending on the distribution of the radiation source and on the target geometry. We performed this estimation by means of an external purposely developed post-processor employing a Monte Carlo approach based on a suitable formulation of the radiative heat transfer problem [15]. The calculation assumes isotropic radiation emission, optically thin plasma [16], perfectly 
absorbing surrounding solid surfaces.

\section{Results and discussions}

It is often found in experiments that the inner target may detach at lower values of $n_{e, O M P}$ or $P_{\text {sep }}$ than the outer one [6], and there is evidence that this behaviour is driven, at least partly, by the action of drifts [17]. As already mentioned in section 3 , we do not include drifts in our simulations, so we expect to find a more balanced divertor than what we would likely observe in the actual experiment. Still, averaging over the available set of simulations we found that about $20 \%$ of the power to the targets go to the inner side, the remaining fraction flowing towards the outer leg. Even in the absence of drifts, we expect that the power crossing the separatrix should enter the SOL preferentially on the outer side [6], and our modelling is consistent with this prediction (on average, we found that $87 \%$ of the power crosses the separatrix on the outer side). To produce a simple estimate, we then assume that all the power enters the SOL at the OMP. An elementary consequence of the 2 points model [6] is:

$$
q_{u} \propto \frac{1}{L}
$$

where $q_{u}$ is the parallel power density flowing upstream along the SOL at the OMP, and $L$ is the connection length to the target. From (1) we can expect the power to split between targets according to the inverse ratio of the connection lengths:

$$
\frac{q_{u, \text { out }}}{q_{u, \text { inn }}}=\frac{L_{\text {inn }}}{L_{\text {out }}}
$$

To evaluate $L_{i n n}$ and $L_{\text {out }}$, we consider a flux tube protruding $5 \mathrm{~mm}$ radially from the separatrix into the SOL, measured at the OMP (this width is larger than the power decay length expected for DEMO following Eich formula [18], and should safely include most of the power flowing to the targets). Averaging across the selected flux tube, we obtain $L_{\text {inn }} \approx 37 \mathrm{~m}$ and $L_{\text {out }} \approx 22 \mathrm{~m}$, from which we would estimate $Q_{\text {inn }} / Q_{\text {out }} \approx 0.37$, about twice the average value calculated over our simulation database $(\approx 0.19)$. Impurity radiation also affects the divertor asymmetry. Ar has an optimal radiation window between approximately $T_{e}=10 \mathrm{eV}$ and $T_{e}=30 \mathrm{eV}$, as can be seen from figure (1). Since the electron temperature at the OMP separatrix is commonly of the order of $100 \mathrm{eV}$, when the $\mathrm{SOL}$ is in the sheath limited regime (corresponding to the lower range of $n_{e, O M P}$ in our set of simulations) $T_{t a r} \approx T_{u}$, and little radiation is produced. At higher upstream densities the target temperature decreases and, when it enters the Ar high radiation window, power losses significantly increase, with a positive feedback on the temperature and energy flux reduction. This process affects primarily the inner target (i.e. the cooler one), strengthening the asymmetry. In the following we will take advantage on this and concentrate our discussion mostly the outer target conditions. We will assume that if we can provide an acceptable operating point for the outer target, the inner one should not raise any additional problem.

We discuss now the possibility to operate the outer divertor in the detached regime, 


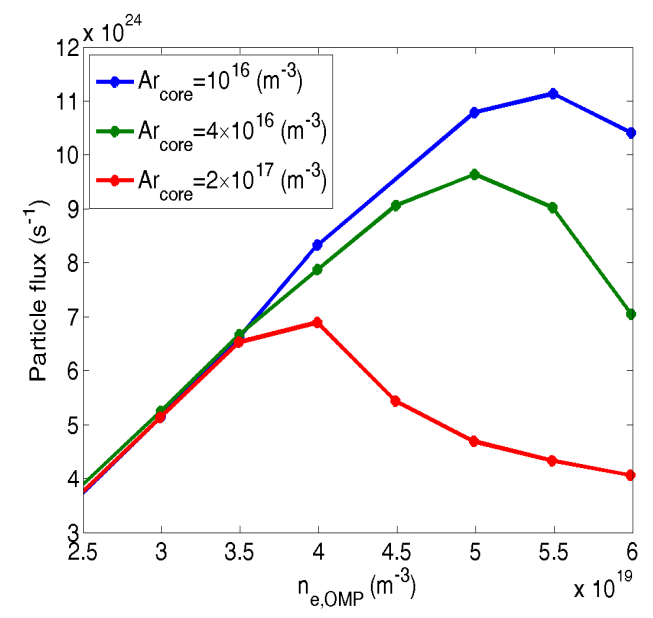

Figure 3. Total $D^{+}$flux at the outer target $(1 / \mathrm{s})$ dependence on $n_{e, O M P}$ and $\mathrm{Ar}$ pedestal density.

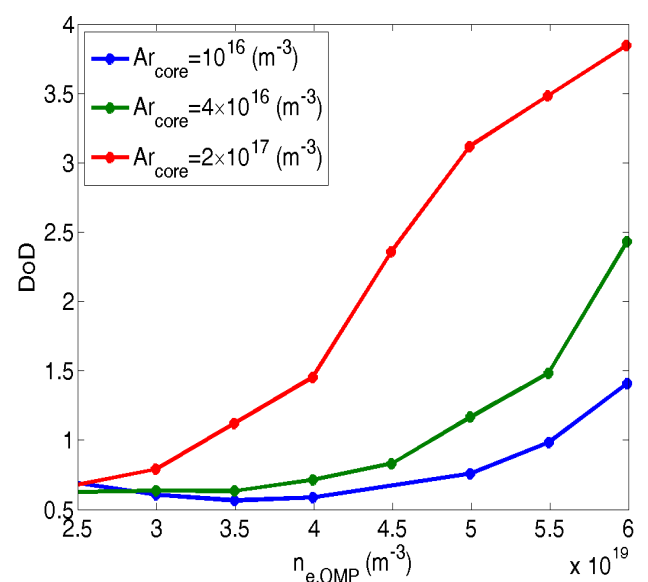

Figure 4. Degree of detachment at the outer strike point, depending on $n_{e, O M P}$ and Ar pedestal density.

which is considered a pre-requisite to allow reducing the power peak load down to tolerable levels. Figure 3 shows the total $D^{+}$particle flux at the outer target as a function of $n_{e, O M P}$ for various pedestal $A r^{18+}$ densities. If we take the roll-over and subsequent progressive reduction of the target particle flux as one of the detachment signatures [6], then we see that for all considered Ar injection levels it is possible to start detaching the outer target, although the flux reduction is sometimes just moderate, which suggests the detachment to be not complete. This is confirmed by figure 4 , which shows the degree of detachment (DoD) evaluated at the outer strike point following eq (19) of [19]. Although the DoD does increase above one for sufficiently large $n_{e, O M P}$, and particularly so for high $A r^{18+}$ core densities, it never gets substantially high $(D o D=\inf$ would correspond to the total plasma disappearance in front of the target, $D o D=10$ may be considered as a full detachment signature [20]). We can notice that for low density values figure 4 reports values $D o D \approx 0.6$, while the simplest version of the 2-Point model would suggest that, in the absence of any momentum loss, DoD should not drop below a minimum value of 1 . However, the 2-Point model assumes all the momentum to be injected at a single upstream location, which we take here to be the OMP, while in the more sophisticated 2D SOLPS model momentum can be transferred from the core plasma into the SOL all along the separatrix, down to the $\mathrm{X}$ point. We take the difference between the 2-Point prediction $D o D_{\min }=1$ and the computed value as a measure of the differences introduced by the more physically comprehensive SOLPS model. Comparison of figures 3 and 4 suggests that, although some detachment level can be obtained at the outer target, this involves a considerable amount of impurities to be injected and a relatively large value of $n_{e, O M P}$, of the order of $\approx 46 \%$ of the Greenwald limit (or higher for the lower impurity values studied). This shows that a possible operational window for DEMO could be relatively narrow. 


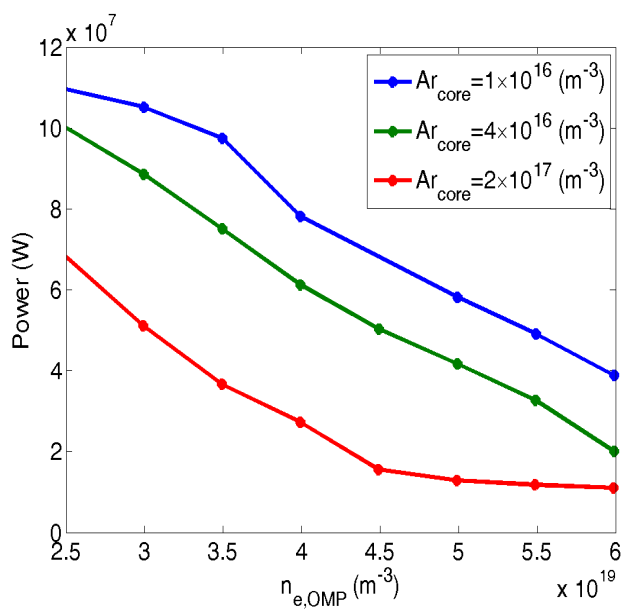

Figure 5. Power at the outer target for three different levels of Ar core density

Figure 5 analyses the effect of $n_{e, O M P}$ and impurity level on the power reaching the outer target. For $n_{A r^{18+}}=10^{16} \mathrm{~m}^{-3}$ or $4 \times 10^{16} \mathrm{~m}^{-3}$ the power to target decreases roughly linearly with $n_{e, O M P}$, and only for the largest level $n_{A r^{18+}}=2 \times 10^{17} \mathrm{~m}^{-3}$ it saturates at $\approx 10 M W$, provided the value of $n_{e, O M P}$ is sufficiently large. This can be understood by looking at figure 1 . Within the range $T_{e}=30-200 \mathrm{eV}$ (which we will show later to cover the characteristic SOL temperatures in most of the cases under consideration) the $A r^{18+}$ radiation rates are slowly, non monotonically, decreasing. If we assume for the sake of simplicity that in this range the radiation rate $\langle\sigma v\rangle_{\text {rad }}$ is roughly constant, then the total radiated power density $\langle\sigma v\rangle_{\text {rad }} n_{A r^{18}+} n_{e}$ becomes proportional to the electron density, which causes the linear decrease of the power to target. When the total dissipated power becomes sufficiently large, the temperature next to the target drops below $10 \mathrm{eV}$, and the radiation rate become quickly negligible (see also figure 7 below). With (almost) no further radiative dissipation taking place, the power to target finally saturates.

The two following figures help to clarify the mechanism through which the presence of impurity influences the target operational regime. They compare the profiles along the separatrix, from the OMP down to the outer strike point, of radiation density (figure 6) and electron temperature (figure 7) for $n_{A r_{18+}}=2 \times 10^{17}\left(\mathrm{~m}^{-3}\right)$ and $n_{e, O M P}=2.5 \times 10^{19} \mathrm{~m}^{-3}$ or $n_{e, O M P}=6.0 \times 10^{19} \mathrm{~m}^{-3}$. The case with higher $n_{e, O M P}$ shows generally larger radiation losses due to the higher electron density, as discussed previously. This results in an overall smaller $T_{e}$. However, until $\approx 5(\mathrm{~m})$ from the target the two $T_{e}$ profiles can be obtained by roughly down-shifting one onto the other. Near the target the two profiles differentiate sensibly: in the high $n_{e, O M P}$ case the temperature is low enough to enter the range $10-30 \mathrm{eV}$, where the radiation rates are maximized. This causes a sharp increase in the radiation losses (clearly visible in figure 6), lasting until the temperature has been pushed down enough to exit from the 


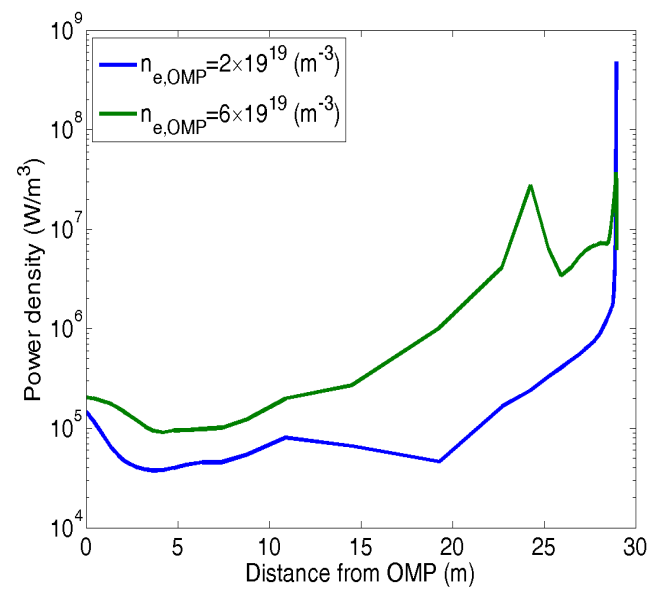

Figure 6. Power density radiated along the separatrix from OMPup to the outer target $\left(W / m^{3}\right)$ for two different $n_{e, O M P}$ levels and Ar pedestal density $2 \times 10^{17}\left(\mathrm{~m}^{-3}\right)$

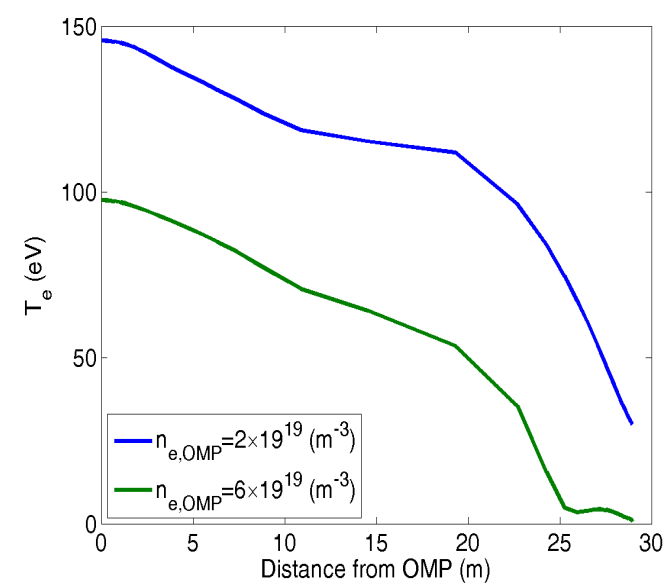

Figure 7. Electron temperature along the separatrix from OMPup to the outer target $\left(W / m^{3}\right)$ for two different $n_{e, O M P}$ levels and Ar pedestal density $2 \times 10^{17}\left(\mathrm{~m}^{-3}\right)$

high-radiation window. At this point, the heat conductivity has dropped sufficiently for the heat flux to be mostly convective, and the temperature profile almost flattens. Both $n_{e, O M P}$ values present a further radiation peak near the target. This is due to $\mathrm{D}$ atoms recycling from the target and ionizing in its proximity as a consequence of the still relatively high temperature value. Radiation from this region is quite intense but extremely localized, so that it does not produce a major contribution to the total radiated power. Still, emitted photons have a high probability to hit the target, and contribute non negligibly to the local power load, as we will discuss later.

We turn now more specifically to the question of target protection. Figure 8 shows the maximum electron temperature along the outer target as a function of $n_{e, O M P}$. The limit of $5 \mathrm{eV}$ is also reported to help evaluating the picture. The curves confirm that getting sufficiently low temperatures for DEMO should be possible, although not easy. The data suggest that sputtering could be limited provided $n_{e, O M P}$ is kept sufficiently large, and a consistent amount of impurity is present in the SOL (at least $47 \%$ of the Greenwald limit for $A r^{18+}$ density $2 \times 10^{17} \mathrm{~m}^{-3}$ ). However, it appears that reducing the peak power level down to tolerable values should be much more difficult. In figure 9 we report the peak power load onto the outer target. The solid lines show the power load accounting only for conduction, convection and potential energy deposition. Dashed lines include also the power deposited by the radiation emitted inside the plasma. Dissipation through radiation is considered the most promising candidate to spread the SOL entering power over a large area, so reducing the peak load onto the divertor targets. However, when a noticeable amount of power is radiated close to the solid structures, the consequent load can still be sharply localized, as already pointed out. In the case with $n_{e, O M P}=6 \times 10^{19} m^{-3} n_{A r^{18+}, \text { core }}=2 \times 10^{17} m^{-3}$, for example, D 


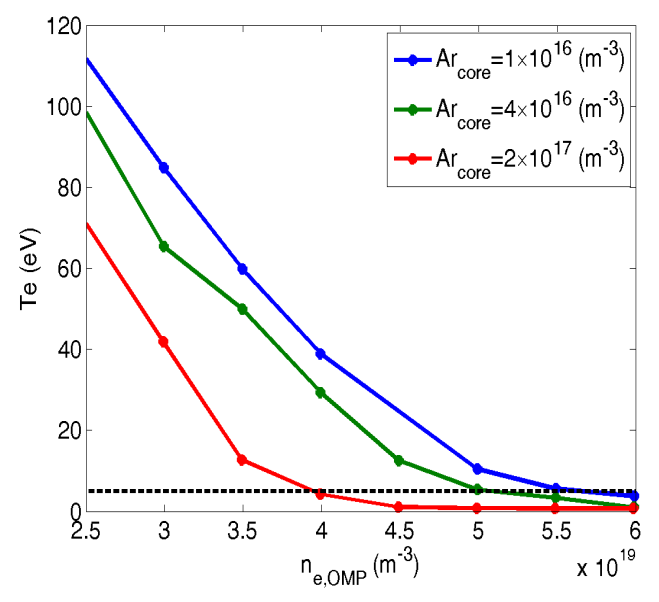

Figure 8. Peak electron temperature along the outer target at three different levels of $A r^{18+}$ core density

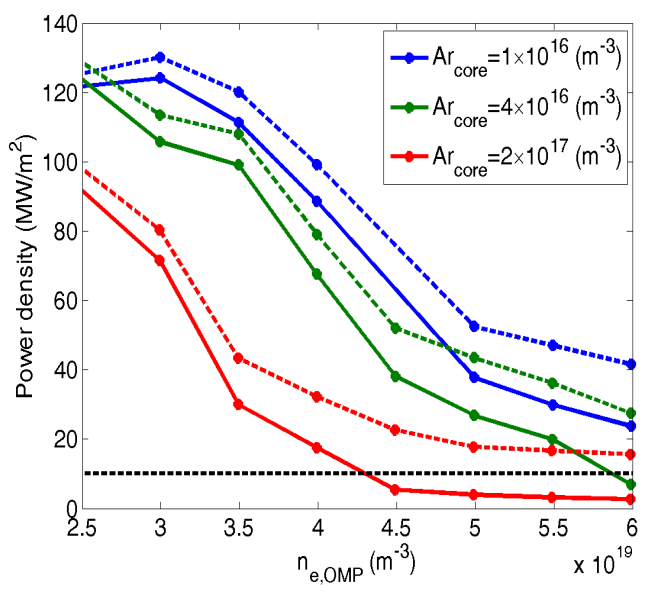

Figure 9. Peak power density along the outer target at three different levels of $A r^{18+}$ core density. Solid lines count only for conduction/advection power deeposition, dashed lines include radiative loads

neutrals entering from the target produce a radiation peak about $2.5 \mathrm{~cm}$ far from the target itself, measured along the magnetic field lines. Taking into account the field lines inclination respect to the solid surfaces, the radiation source is extremely close to the target itself. In contrast to this result, it is found experimentally that when all metal machines operate in full detachment a large fraction of the SOL radiation tends to originate near the $\mathrm{X}$ point region [2], and our modelling suggests that, for a sufficiently large $D o D$, every radiation region should actually migrate upstream along the separatrix. If DEMO radiated mostly from the $\mathrm{X}$ point, rather than very close to the target surface, a positive effect on the peak power load should be obtained because the emitted light would distribute more uniformly over a relatively larger area. It is then worth discussing briefly if such condition could be obtained in DEMO.

A major obvious difference between the observations reported in [2] and the ones we are describing is that the development of a stable region of strong $\mathrm{X}$ point radiation is associated with operation in fully detached regime, while we were able to obtain only moderate $D o D$ levels. A complete analysis of possible strategies to drive DEMO towards full detachment may involve more sophisticated considerations, including for example a better description of the neutral physics (possible only if the neutrals are modelled kinetically), or the analysis of different wall locations to puff the impurities from. This is beyond the scope of the present paper; however, i $\mathrm{n}$ order to obtain a qualitative check of the possible effect of higher $D o D$ on the radiation distribution we modified the case with $n_{e, O M P}=6 \times 10^{19} \mathrm{~m}^{-3}, n_{A r^{18+}, \text { core }}=2 \times 10^{17} \mathrm{~m}^{-3}$ by reducing progressively the power crossing the separatrix to 120 and $90 \mathrm{MW}$, forcing the plasma 


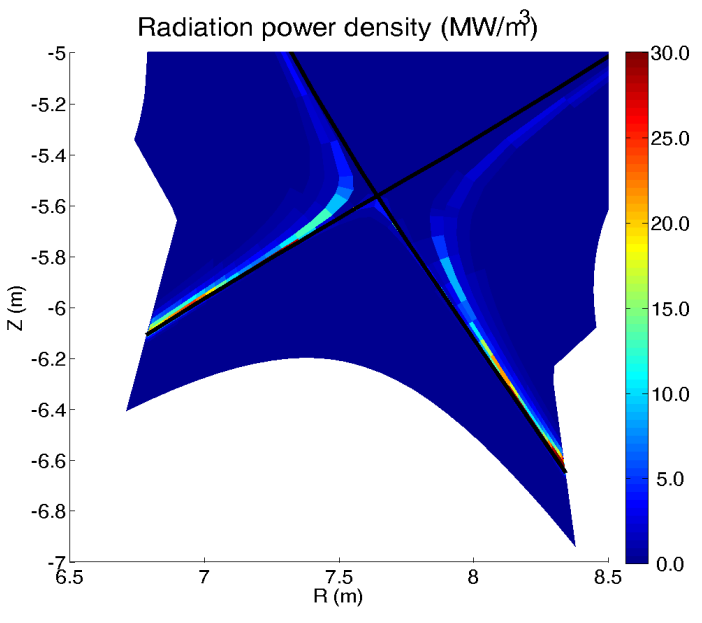

Figure 10. Radiation density from the divertor region for case $n_{e, O M P}=6 \times 10^{19} \mathrm{~m}^{-3}$, $n_{\text {Ar } 18+, \text { core }}=2 \times 10^{17} \mathrm{~m}^{-3}$ at full $P_{\text {sep }}$

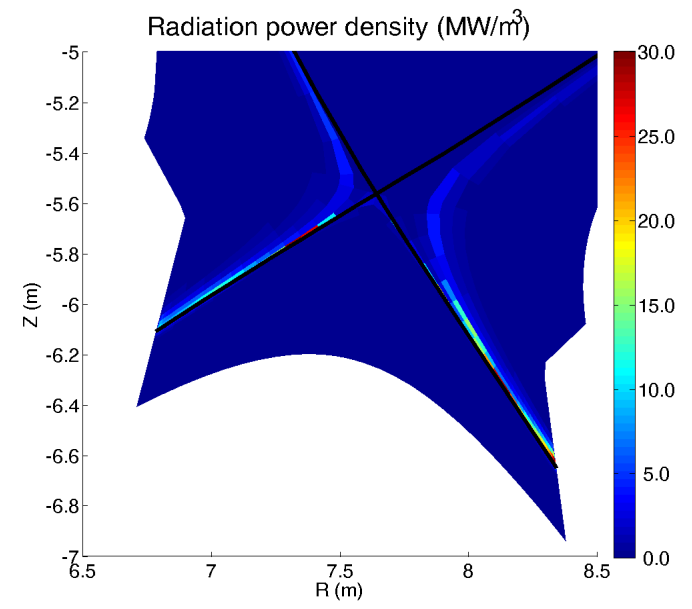

Figure 11. Radiation density from the divertor region for case $n_{e, O M P}=6 \times 10^{19} \mathrm{~m}^{-3}$,

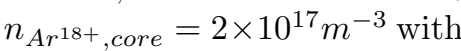
$P_{\text {sep }}$ reduced to $120 \mathrm{MW}$

to detach. The result of this experiment are shown in figures $10-12$ below. They show a 2D map of the radiation density observed in the divertor region for the reference case $\left(P_{\text {sep }}=150 \mathrm{MW}, \mathrm{DoD}=3.8\right.$ at the outer target), an intermediate case with $P_{\text {sep }}=120 \mathrm{MW}(D o D=4.6)$ and an extreme case with $P_{\text {sep }}=90 \mathrm{MW}\left(D_{o} D=6.8\right)$. In all cases we observe radiation coming from the flux tubes closest to the separatrix. At full power, figure 10, the emission is concentrated, on the outer side, adjacent to the target, while on the inner side the peak is a bit upper along the separatrix. At medium power, figure 11, radiation from the inner side moved noticeably towards the $\mathrm{X}$ point, while a still noticeable radiation peak is attached to the outer target. At the minimum power level, figure 12, very little radiation is coming from the inner target, only a moderate amount from the outer one and a strong peak has developed near the $\mathrm{X}$ point. Although there are differences with respect to the development of the $\mathrm{X}$ point radiation region as described in [2] (for example, in that case the fully developed region lies inside the confined plasma while we have it still out from the separatrix), the evolution we observed, with the radiation region moving to the $\mathrm{X}$ point along the inner separatrix first and the outer side following later, is qualitatively consistent with that observed experimentally. This suggests that, should it be possible to completely detach the divertor in DEMO, the development of an $\mathrm{X}$ point radiation region would be likely, with possibly beneficial effects on the target peak load.

\section{Conclusions}

In this paper we produced a first analysis of the possibility to employ a purposely seeded impurity radiator to reduce the power to divertor in the EU-DEMO down to tolerable levels. For this initial analysis, Ar was selected as the adopted impurity, 


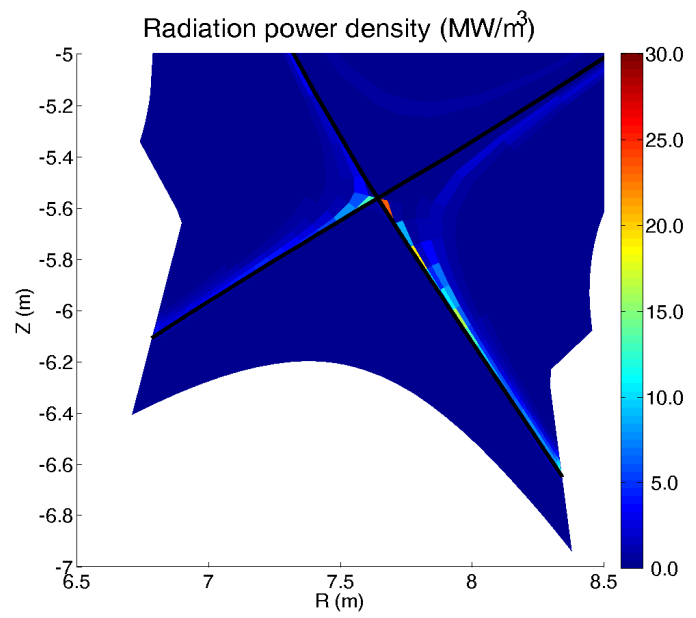

Figure 12. Radiation density from the divertor region for case $n_{e, O M P}=6 \times 10^{19} \mathrm{~m}^{-3}$, $n_{A r^{18+}, \text { core }}=2 \times 10^{17} \mathrm{~m}^{-3}$ with $P_{\text {sep }}$ reduced to $90 \mathrm{MW}$

to take advantage of its radiation window at SOL characteristic temperatures. It has been shown that a proper amount of impurity injection can reduce the plasma temperature in front of the target to levels at which $\mathrm{W}$ sputtering should not be a major problem. However, a relatively large upstream density is required, which can dangerously approach the Greenwald limit. On the other hand, it seems difficult to limit the peak power to the target, due to regions of strong radiation developing just in front of the solid structures, which can generate concentrated spots of high power load. Experiments on current machines predict, and our modelling partially confirms, that if full detachment is reached the large radiation source should move along the separatrix up to the $\mathrm{X}$ point. If this is confirmed, reduced target proximity of the photons source could be beneficial. However, this effect seems to be associated with full divertor detachment, which was not obtained in our simulations. This would probably require a more detailed physics of neutral particles (i.e. the more complete but time consuming kinetic model), a better description of the divertor geometrical structures and a careful choice of the impurity injection location. All of this is likely to help understanding under which conditions the EU-DEMO divertor will be able to detach completely; nonetheless our modelling suggests that the acceptable operational window is likely to be narrow.

In the present analysis, for the sake of simplicity, we did not consider the details of coupling with the core plasma. By always setting $P_{\text {sep }}=150 \mathrm{MW}$ we implicitly assumed that a large amount of power should be radiated within the pedestal. It is unlikely that Ar could be the right radiator in the confined plasma, so that at least another impurity should probably be injected. Also, we did not evaluate if the impurity levels assumed for the SOL are still compatible with a possible excessive accumulation in the core. A more detailed description of the way impurities enter the plasma and 
distribute between core and SOL, the consideration of one (or possibly more) impurity $\operatorname{mix}(\mathrm{es})$ and compatibility with the core plasma are the first obvious extensions of this work. Such analysis is currently in progress and will be presented in the future.

\section{Acknowledgement}

The authors wish to thank Dr. Thomas Eich for help on discussing the possible range of the separatrix density limit in DEMO. This work has been carried out within the framework of the EUROfusion Consortium and has received funding from the Euratom research and training programme 2014-2018 under grant agreement No 633053. The views and opinions expressed herein do not necessarily reflect those of the European Commission.

\section{References}

[1] Romanelli F 2012 Fusion electricity - a roadmap to the realization of fusion energy Tech. rep. EFDA

[2] Reimold F, Wischmeier M, Bernert M, Potzel S, Kallenbach A, Mller H, Sieglin B and Stroth U 2015 Nucl. Fusion 55033004 URL http://dx.doi.org/10.1088/0029-5515/55/3/033004

[3] Wenninger R, Bernert M, Eich T, Fable E, Federici G, Kallenbach A, Loarte A, Lowry C, McDonald D, Neu R, Ptterich T, Schneider P, Sieglin B, Strohmayer G, Reimold F and Wischmeier M 2014 Nucl. Fusion 54114003 URL http://dx.doi.org/10.1088/0029-5515/54/11/114003

[4] Wenninger R, Arbeiter F, Aubert J, Aho-Mantila L, Albanese R, Ambrosino R, Angioni C, Artaud J F, Bernert M, Fable E, Fasoli A, Federici G, Garcia J, Giruzzi G, Jenko F, Maget P, Mattei M, Maviglia F, Poli E, Ramogida G, Reux C, Schneider M, Sieglin B, Villone F, Wischmeier M and Zohm H 2015 Nuclear Fusion 55063003

[5] Martin Y R, Takizuka T and the ITPA CDBM H-mode Threshold Data Group 2008 J. Phys.: Conf. Ser. 123 012033 URL http://dx.doi.org/10.1088/1742-6596/123/1/012033

[6] Stangeby P C 2000 The Plasma Boundary of Magnetic Fusion Devices (Institute of Physics)

[7] Kallenbach A, Sun H, Carralero D, Eich T, Hobirk J, Scarabosio A, Siccinio M, team A U and team M Parameter dependences of the separatrix density in nitrogen seeded asdex upgrade h-mode discharges 44th EPS Conference, Belfast, Northern Ireland

[8] Schneider R, Bonnin X, Borrass K, Coster D, Kastelewicz H, Reiter D, Rozhansky V and Braams B 2006 Contributions to Plasma Physics 463 - 191 ISSN 0863-1042

[9] Subba F 2016 Simulations of sol plasmas in demo Tech. rep. EUROFusion URL https: //idm. euro-fusion.org/?uid=2MS27P

[10] Stangeby P and Chankin A 1996 Nuclear Fusion 36 839-852

[11] Bonnin X and Coster D 2011 Journal of Nuclear Materials 415 S488-S491 URL http://dx.doi.org/10.1016/j.jnucmat.2010.10.041

[12] Coster D P 2016 Contributions to Plasma Physics URL http://dx.doi.org/10.1002/ctpp. 201610035

[13] Roy C 2005 Journal of Computational Physics 205 131-156

[14] Subba F 2016 Final report on deliverable solps simulations of plasmas in demo Tech. rep. Eurofusion URL https://idm. euro-fusion.org/?uid=2LM2VP

[15] Modest M 2003 Radiative Heat Transfer (Elsevier)

[16] Freidberg J P Plasma Physics and Fusion Energy (Cambridge University Press)

[17] Pitts R, , Andrew P, Bonnin X, Chankin A, Corre Y, Corrigan G, Coster D, Duran I, Eich T, Erents S, Fundamenski W, Huber A, Jachmich S, Kirnev G, Lehnen M, Lomas P, Loarte A, 
Matthews G, Rapp J, Silva C, Stamp M, Strachan J and Tsitrone E 2005 Journal of Nuclear Materials 337-339 146-153

[18] Eich T, Sieglin B, Scarabosio A, Fundamenski W, Goldston R J and and A H 2011 Physical Review Letters 107

[19] Siccinio M, Fable E, Lackner K, Scarabosio A, Wenninger R P and Zohm H 2016 Plasma Physics and Controlled Fusion $\mathbf{5 8} 125011$

[20] Loarte A 1988 Nuclear Fusion 38 331-371 